\title{
MODIS Solar Diffuser On-orbit Degradation Characterization Using Improved SDSM Screen Modeling
}

\author{
H. Chen ${ }^{\mathrm{a}}$, X. Xiong ${ }^{\mathrm{b}}$ A. Angal ${ }^{\mathrm{a}}$, Z. Wang ${ }^{\mathrm{a}}$ and A. Wu ${ }^{\mathrm{a}}$ \\ ${ }^{a}$ Science Systems and Applications, Inc., 10210 Greenbelt Road, Lanham, MD 20706; \\ ${ }^{\mathrm{b} S}$ Science Systems and Exploration Directorate, NASA/GSFC, Greenbelt, MD 20771
}

\begin{abstract}
The Solar Diffuser (SD) is used for the MODIS reflective solar bands (RSB) calibration. An on-board Solar Diffuser Stability Monitor (SDSM) tracks the degradation of its on-orbit bi-directional reflectance factor (BRF). To best match the SDSM detector signals from its Sun view and SD view, a fixed attenuation screen is placed in its Sun view path, where the responses show ripples up to $10 \%$, much larger than design expectation. Algorithms have been developed since the mission beginning to mitigate the impacts of these ripples. In recent years, a look-up-table (LUT) based approach has been implemented to account for these ripples. The LUT modeling of the elevation and azimuth angles is constructed from the detector 9 (D9) of SDSM observations in the MODIS early mission. The response of other detectors is normalized to D9 to reduce the ripples observed in the sun-view data. The accuracy of all detectors degradation estimation depends on how well the D9 approximated. After multiple years of operation (Terra: 16 years; Aqua: 14 years), degradation behavior of all detectors can be monitored by their own. This paper revisits the LUT modeling and proposes a dynamic scheme to build a LUT independently for each detector. Further refinement in the Sun view screen characterization will be highlighted to ensure the degradation estimation accuracy. Results of both Terra and Aqua SD on-orbit degradation are derived from the improved modeling and curve fitting strategy.
\end{abstract}

Keywords: MODIS, solar diffuser, solar diffuser stability monitor, calibration, radiometer

\section{INTRODUCTION}

MODIS on Terra and Aqua was launched on December 18, 1999 and May 4, 2002, respectively. It is a cross-track scanning radiometer with 36 spectral bands, including reflective solar bands (RSB) with wavelength ranging from 0.412 to $2.2 \mu \mathrm{m}$ (Bands 1-19 and 26), and thermal emissive bands (TEB) with wavelength ranging from 3.7 to $14.5 \mu \mathrm{m}$ (Bands 20-25 and 27-36). The observations are made at three spatial resolutions (nadir): $0.25 \mathrm{~km}$ for Bands $1-2$ with 40 detectors per band, $0.5 \mathrm{~km}$ for Bands 3-7 with 20 detectors per band, and $1 \mathrm{~km}$ for Bands 8-36 with 10 detectors per band [1]. All the detectors are distributed on four focal plane assemblies (FPA) according to their wavelengths: visible (VIS), near infrared (NIR), short- and mid-wave infrared (SMIR), and long-wave infrared (LWIR). In order to monitor and maintain the sensor calibrated data quality, it was designed with a set of on-board calibration subsystems, including a solar diffuser (SD), a solar diffuser stability monitor (SDSM), a spectroradiometric calibration assembly (SRCA), and a v-grooved blackbody (BB). The SRCA is primarily used for the sensor's spectral (RSB only) and spatial (TEB and RSB) characterization. The SD and SDSM are normally used together for RSB calibration and the BB for TEB calibration. There is a space view (SV), an aperture providing a view of cold space, to allow for a zero radiance input signal on each scan. This paper focuses on MODIS Aqua and Terra SDSM and presents their on-orbit performance.

The MODIS SD bi-directional reflectance factor (BRF) was characterized pre-launch. Its on-orbit changes are monitored by the on-board SDSM. Functionally, the SDSM is operated during each SD calibration to obtain alternate views of the sunlight through a fixed attenuation screen and that reflected from the SD panel. This fixed screen is a metal screen with uniformly distributed pinhole arrays on it. Thus, its transmission function oscillates with the solar angles, creating "ripples" as a function of solar azimuth and elevation angles. The two viewing paths are termed as the Sun view and the SD view, 
respectively. Due to the misalignment of the screen, the ratio shows significant variations depending on the view geometry of the solar illumination with oscillations as large as 10\% [2]. The impact needs to be mitigated in order to make a reliable estimation of the SD's degradation. More details about the SD and SDSM are discussed in [3], where a brief review on SDSM function and operations has been presented. In this paper, estimation models for SD degradation are addressed, including a regular approach of absolute ratio between the response of Sun and SD views, a normalization approach to mitigate the impacts due to screen misalignment [2,3], and a look-up table (LUT) based approach for characterizing the degradation of SDSM detector 9 (D9) [4].

Since the observed ripples were common to all detectors, an alternative methodology was developed to track the SD degradation by normalizing other SDSM detectors to D9, with the assumption that the SD degradation at the wavelength of $0.936 \mu \mathrm{m}$ (SDSM D9) is relatively small. Using the normalization approach, a stable time-series of SD/Sun response was obtained to effectively track the SD degradation. After a decade of successful performance of the SD degradation estimation using the alternative methodology, the accumulated degradation at the SDSM D9 wavelength became noticeable and needed to be corrected. However, the direct ratio of SD/Sun response for D9 still presented the challenges associated with the screen misalignment. Therefore, instead of direct ratio of responses from the SD and Sun views, a LUT-based correction is applied. The LUT is derived using the datasets from early part of the mission when the SDSM D9 degradation was observed to be negligible.

In this paper, we revisit the LUT modeling and propose to build LUT independently for each SDSM detector. Improvements in the current technique are also investigated. Further refinement in the Sun view screen characterization is also discussed. An overall performance of the SD degradation for both instruments is also discussed.

\section{SDSM SUN-VIEW SCREEN MODELING}

\subsection{Dynamic Model for Individual Detectors}

MODIS SD/SDSM calibration is performed periodically to track the on-orbit detector gain change for the RSB. Recall that there are nine filtered detectors in the SDSM covering wavelengths from 412 to $936 \mathrm{~nm}$ to track the on-orbit SD BRF changes. During each SD calibration event, the impact due to the attenuation screen is dependent on the view-geometry. Paper [1] summarized different degradation estimation approaches including: (i) traditional approach (absolute ratios); (ii) relative approach (normalized to detector 9); (iii) look-up-table based correction approach. The absolute ratios of the SD view response to the Sun view response are normalized to the first measurement. The time series of these ratios for each SDSM detector determines the SD degradation. In the relative approach, the ratios are also normalized to D9 so as to remove the ripples and produce a stable time series to track the SD degradation. The SD degradation is negligible at the wavelength of D9, especially in the early mission period. A noticeable degradation of the SDSM D9 has been observed over the decade of MODIS operations. An additional correction of SDSM D9 is essential for accurate estimation of SD degradation at all MODIS wavelengths. To better characterize MODIS SD on-orbit degradation and to support MODIS Level 1B (L1B) calibration for the collection 6 (C6) reprocessing, tradeoff studies have been conducted to generate a D9 LUT, where the time independent geometrical features of the attenuation screen have been characterized. With the LUT based approach, a more reliable estimate of SD degradation at D9 wavelength can be achieved.

As an example, Figure 1 plots Aqua SDSM Sun view responses on August 31, 2002. All SDSM detectors show a similar fluctuation feature. However, their responses (digital counts) are obviously different in magnitude. Thus, simply relative to D9, it is unavoidable to introduce degradation estimation error for other detectors. In this paper, we propose an enhanced method to build a LUT for each individual detector. Paper [1] has discussed a LUT-based approach, where a correction factor was developed based on the SDSM D9 response as a function of elevation and azimuth angle. Here we extend this idea to other SDSM detectors. All datasets include both SD/Sun view responses and their corresponding elevation and azimuth angles $(\alpha, \beta)$. Then, an equally spaced two-dimensional bin of $(\alpha, \beta)$ within a pre-defined LUT size was generated. After that, a LUT for detector $\mathrm{k}, \Lambda^{k}(\alpha, \beta)$, is associated with $\varpi(\alpha)$ and $\varpi(\beta)$, which are group sets of digitalized $(\alpha, \beta)$ angles. Assume that $\left(a_{i}, b_{j}\right)$ denotes jointly $(i, j)$ bin indexes, then we have, 


$$
{ }^{k}\left(a_{i}, b_{j}\right)=\left\langle\frac{d c_{S U N}^{k}\left(a_{i}, b_{j}\right)}{d c_{S D}^{k}\left(a_{i}, b_{j}\right)} \times{ }^{k}\right\rangle \quad ; \quad a_{i} \quad(\quad), b_{j} \quad(), k=1,2, \square, 9 .
$$

where $\eta^{k}$ denotes the reciprocal of degradation on detector $k$ at the time SDSM data considered. This ratio reflects the impact of the solar geometry to the SDSM screen transmission alone. A spline fitting over elevation and azimuth dimensions, as well as a smooth processing, is performed to generate an interpolated value of the LUT. With the ratio LUT, the SDSM detector degradation can be calculated by

$$
\Delta_{S D}^{k}=\left\langle\frac{d c_{S D}^{k}(\alpha, \beta)}{d c_{S U N}^{k}(\alpha, \beta)} \cdot \Lambda^{k}(\alpha, \beta)\right\rangle ; k=1,2, \ldots, 9 .
$$

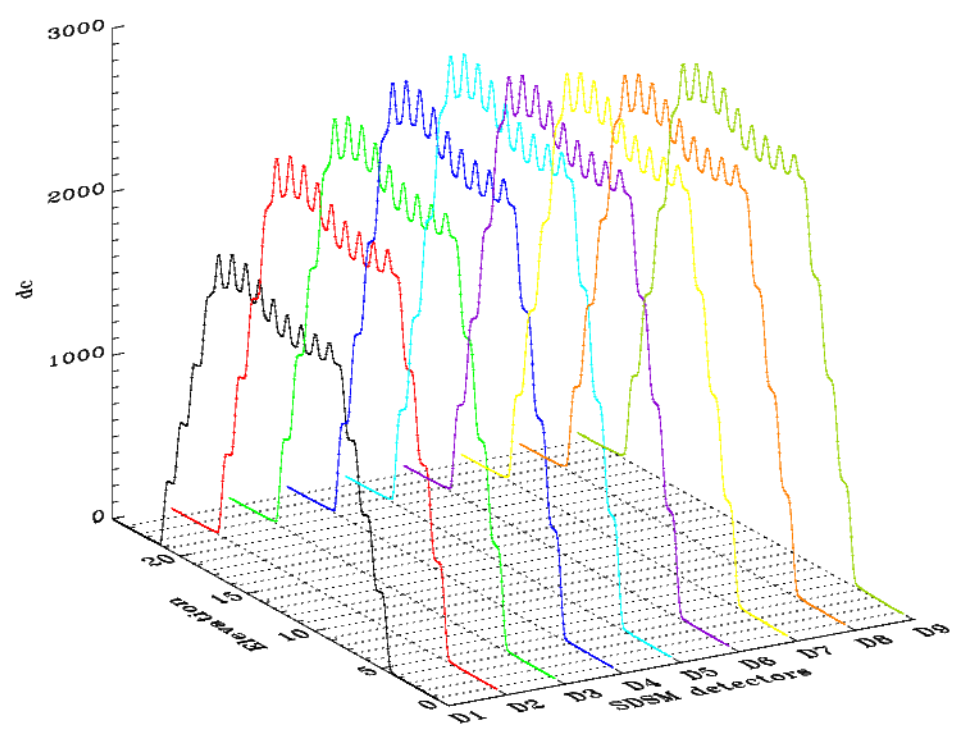

Figure 1. Aqua SDSM Sun view response @ 2002243 (August 31, 2002).

The uncertainty of SD degradation estimation is decided by the accuracy of the LUT generation. The degradation observed by other SDSM detectors far exceeds the degradation observed by SDSM D9. Since the per-detector LUT is generated using the first few years of SD and Sun observations, the degradation of the SD in this time-period cannot be ignored. In order to mitigate this effort, a modified approach (dynamic LUT approach) has been formulated. At first, SDSM raw data are corrected using the initial screen compensation LUT, where the previously generated D9-based LUT can be used as an example. Then, degradation estimation is performed for each detector. The degradation coefficient of each detector will be re-applied as a part of the de-trending process. After de-trending, the processed SDSM data will be used for each individual detector LUT re-generation. Each detector's degradation is then re-estimated using this re-generated LUT. The dynamic routine will be stopped while the degradation estimation is stable or fitting residue of processed data sets is unchanged. Because each SD/SDSM calibration is performed at a discrete time, the measurements are sparse and the LUT generated is not accurate enough to calculate the SD degradation for any single calibration. Proper data processing techniques have to be applied. In practice, dynamic results show that five iterations are good enough to obtain a stable estimate for the SDSM screen characterization.

\subsection{Aqua MODIS SDSM Sun-view Screen LUT}

The first five years of SDSM "alternative" mode measurements have been used to derive the LUT based on elevation and azimuth angles. To eliminate the uncertainty impact of the Solar Diffuser Screen (SDS), data acquired with the SDS open are used to build the LUT for Aqua. Figure 2 shows the Aqua D9 LUT and deference contours of all other detectors relative 
to it. The three dimensional LUT profile is plotted for detector 9 only and the results for detectors are shown the LUT difference expressed by,

$$
\delta_{k}=\Lambda^{k}(\alpha, \beta)-\Lambda^{9}(\alpha, \beta) ; k=1,2, \ldots, 8 .
$$

Aqua D9 LUT surface profile plotted as a function of elevation and azimuth angles shows fluctuations that are reflective of the SDSM screen's attenuation. In this figure, the deference is presented in a color bar, which is shown in the right lower corner. From the comparison results, a difference of up to $5 \%$ is observed in D1. Based on the color bar, the three LUTs of D6, D7, D8 are closely matched the D9 LUT.

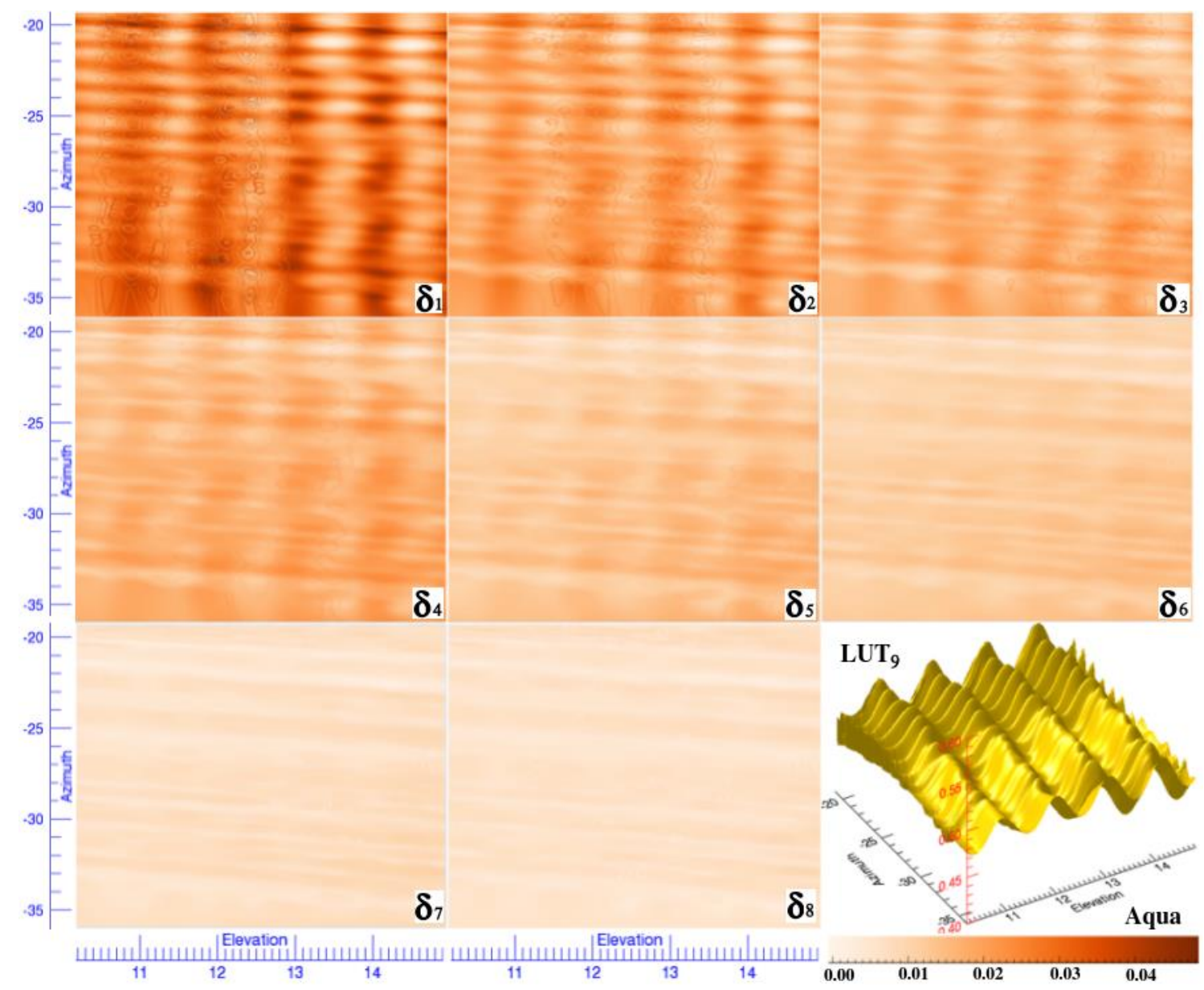

Figure 2. Aqua LUT comparisons for all nine detectors. 

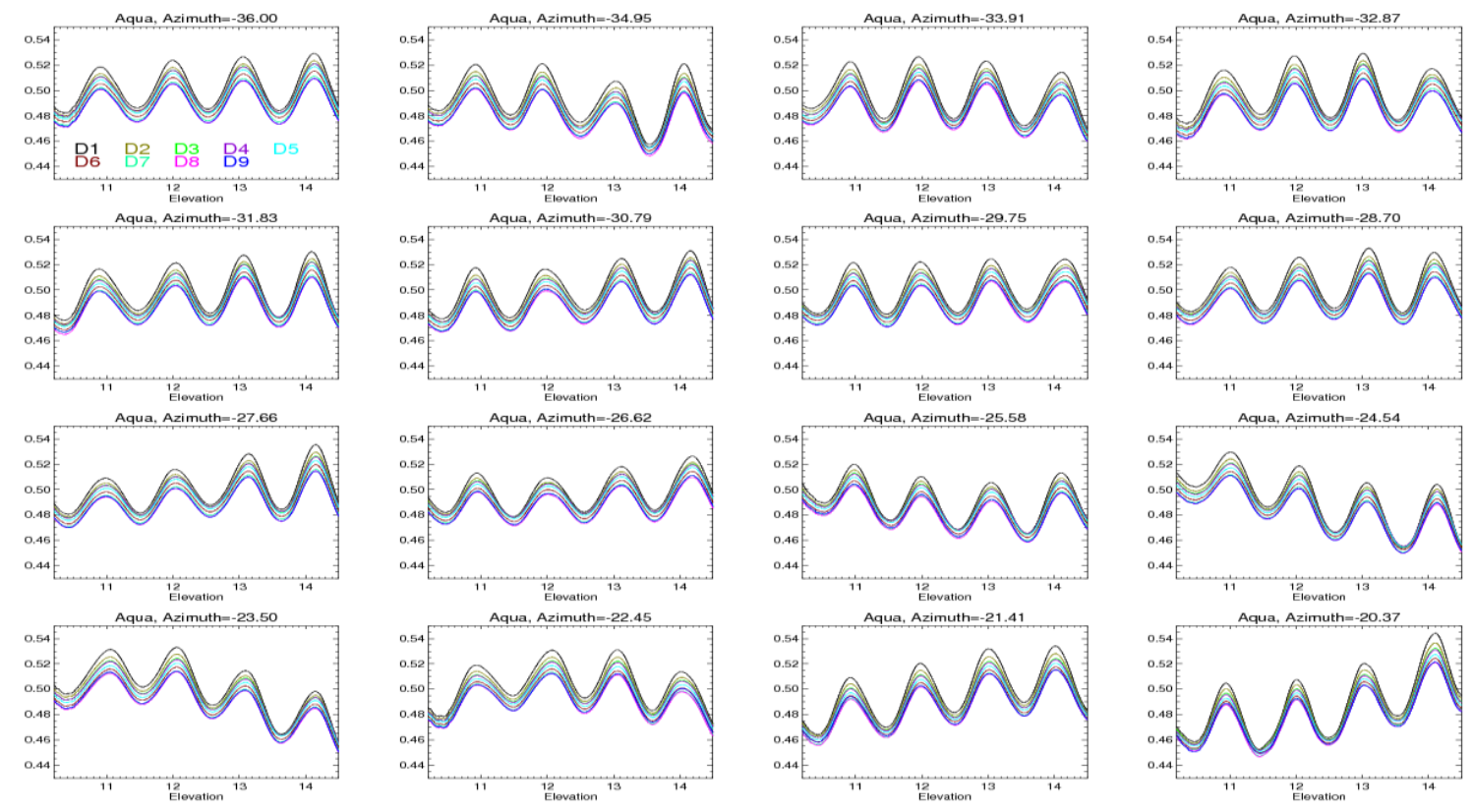

Figure 3. Aqua SDSM pin-hole screen elevation profiles for all detectors.

To present the LUT feature associate with elevation angles, Figure 3 plots Aqua all detector LUTs for different azimuth angles in a range of $-20.37^{\circ}$ to $-36.00^{\circ}$. Based on the elevation profiles in different cases of azimuth angles, the LUT feature has different behavior.

\subsection{Terra MODIS SDSM Sun-view Screen LUT}

In the case of MODIS Terra, there are two distinctive trends in the SD degradation: before and after the July 2003 SD door (SDD) anomaly [2-4]. After the door anomaly, the SDD is in a fixed open position and the SDS is permanently in place so that all the RSB are always calibrated using an attenuated solar response. As a result of this operational change, Terra MODIS RSB calibration coefficients can be monitored every orbit. The degradation rates have accelerated after this anomaly, and are strongly wavelength dependent with larger degradation observed at short wavelengths. After the anomaly, the SDSM calibration is only available with the SDS in place. For consistency, only SDS close mode data are used to build the LUT. In practice, the first three years measurements (before 2003 SDD anomaly) are used to build an initial LUT for each detector, and dynamic iterations on the 5-year data after the SDD anomaly are performed. Similar to the case of Aqua MODIS, Figure 4 shows the Terra D9 LUT surface profile and difference contours of all other detectors relative to it. The difference is in the range of $-12 \%$ to $5 \%$, which is larger than Aqua MODIS. The reason is mainly due to the fact that Aqua uses SDS "open-mode" data and Terra using SDS "closed-mode" data, which have more noise. Figure 5 plots Terra all detector LUTs for different azimuth angles in a range of $-16.15^{\circ}$ to $-32.89^{\circ}$. Based on the elevation profiles in different cases of azimuth angles, the LUT feature has different behavior as well. 


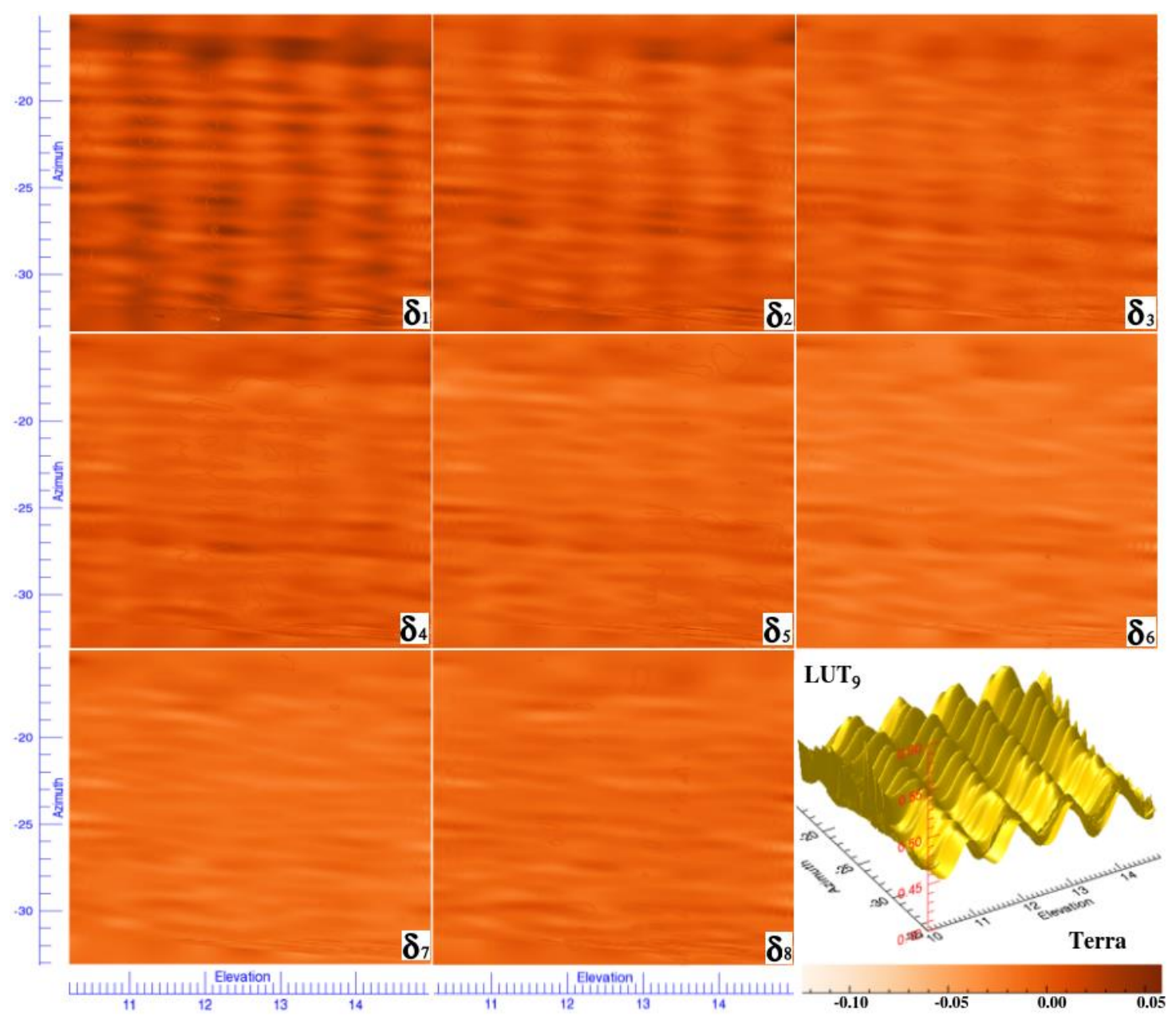

Figure 4. Terra LUT comparisons for all nine detectors.
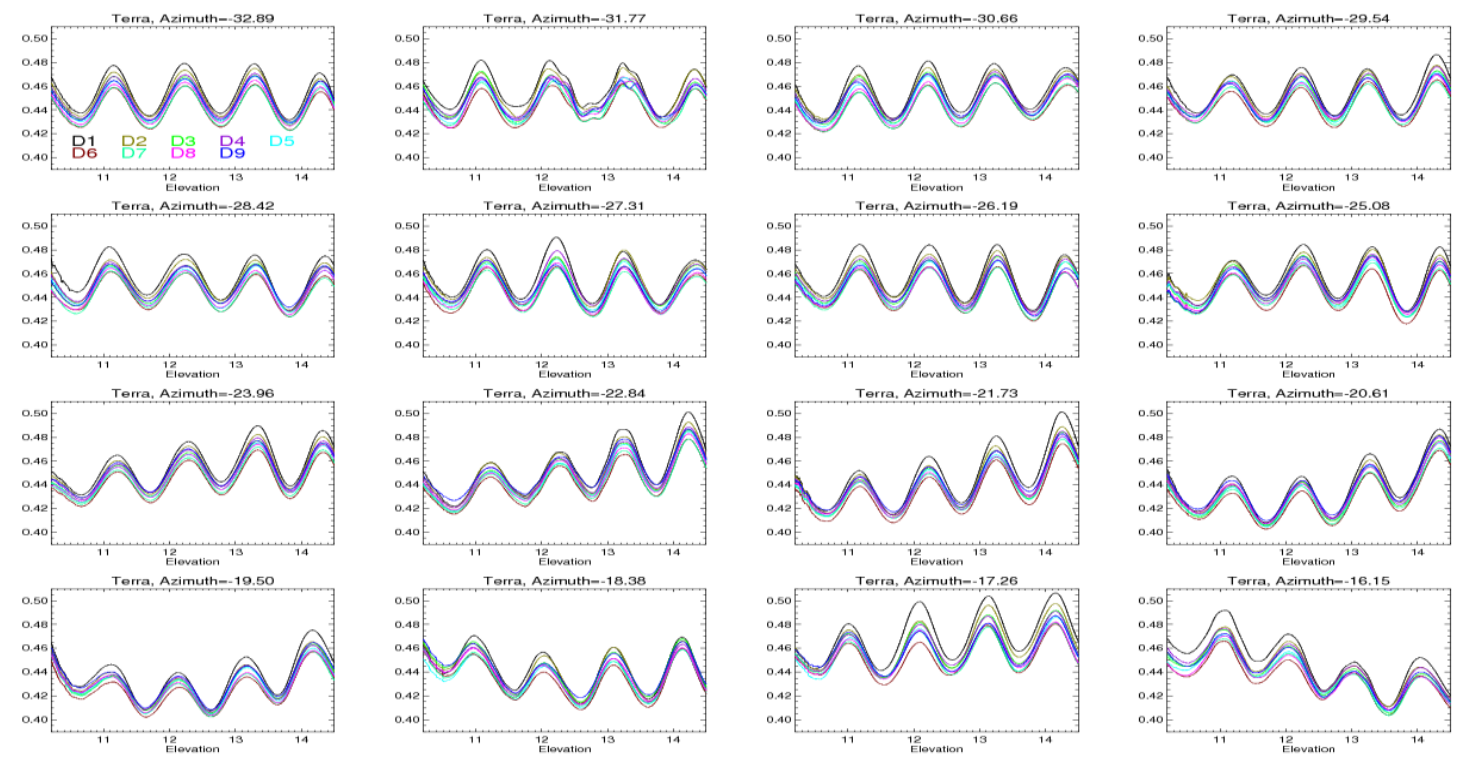

Figure 5 Terra SDSM pin-hole screen elevation profiles for all detectors. 


\section{ESTIMATION RESULTS}

In order to prolong the operational lifetime of the SD and SDSM, the frequency of the calibration activities have been gradually reduced. In the early mission time, SD/SDSM calibration activity was performed on a weekly basis to track SD BRF change. Over time, the frequency was reduced to two weeks and subsequently to three weeks, which is the current operational configuration. Now, the SDSM is operated with SDS open configuration once every six weeks for Aqua MODIS, although the SDSM operation still remains on a tri-weekly basis. Fortunately, the SD degradation does not exhibit any dependence on the SD/SDSM calibration frequency. The reason is that in addition to RSB SD calibration, the SD also degrades due to exposure to Earthshine through the nadir port during the daylight part of each orbit. Data analysis [7] shows that nadir earthshine dominates the SD degradation, especially for the three short-wavelength MODIS bands, band$8(0.412 \mu \mathrm{m})$, band-9 $(0.443 \mu \mathrm{m})$ and band-3 $(0.469 \mu \mathrm{m})$.

As presented in the previous section, a more reliable SD degradation can be derived if the Sun view screen pinhole effects are characterized by using the dynamic model for all SDSM detectors. Although the LUT is applied to all the SDSM detectors, only the results from select detectors is presented for clarity. A comparison of three datasets are presented in the two charts: (i) raw ratios of measurements in the SUN view and the SD view; (ii) corrected ratios using a D9-based LUT, which is currently employed in the MODIS mission operations; (iii) corrected ratios using the newly proposed dynamic individual LUTs. In the case (ii), the D9-based LUT is regularly built using the first-three year's data without de-trending considerations, as D9 degradation is ignorable in the first three years. All SDSM detectors use the same D9 LUT. In the current operations, the corrected ratios are further processed by using a sliding-window average (SWA). Based on Figure 6 , we can observe that the case of using the dynamic individual LUTs provides the best result, where much smaller fluctuations in the data trending can be achieved. In addition, using the SWA is not necessary as the data behavior is sufficiently stable. Detectors operated at shorter wavelengths, such as D1 and D2, show larger SD degradation. All SDSM detectors use the same fitting functions as in case (iii), where the effectiveness of the geometrical correction is proved in a long-term period. Both Terra and Aqua responses are expected at a similar uncertainty level.
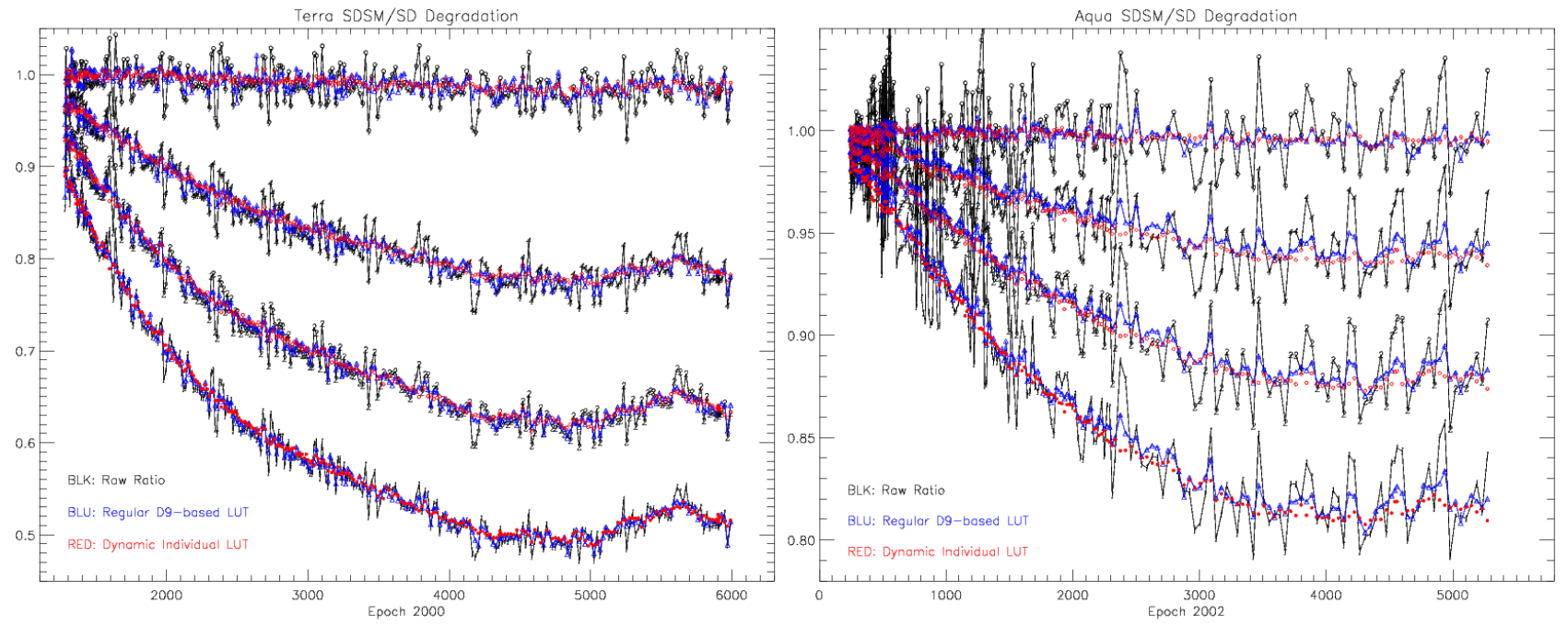

Figure 6 Terra and Aqua SDSM performance trending of four detectors (D1, D2, D4, D9).

Figure 7 plots Terra and Aqua MODIS degradation trending results, where all SDSM detectors of each instrument use the same fitting strategy. Notice that the Aqua MODIS SDSM degradation rates are nearly identical to Terra when both instruments are operated under same conditions. Thus, in the first three operation years, Aqua and Terra have similar degradation profiles. Since Terra MODIS experienced a SDD anomaly, the later degradation profiles show much larger change compared to Aqua MODIS, especially for shorter wavelengths. Also, it was found that in 2014-2015, that the SD degradation trending reverse direction for a brief period, which was unexpected. The reason for this change in the trend is currently unclear. We will continue to monitor this behavior. In the Terra chart, two different degradation characteristics before and after the anomaly are clearly presented. 

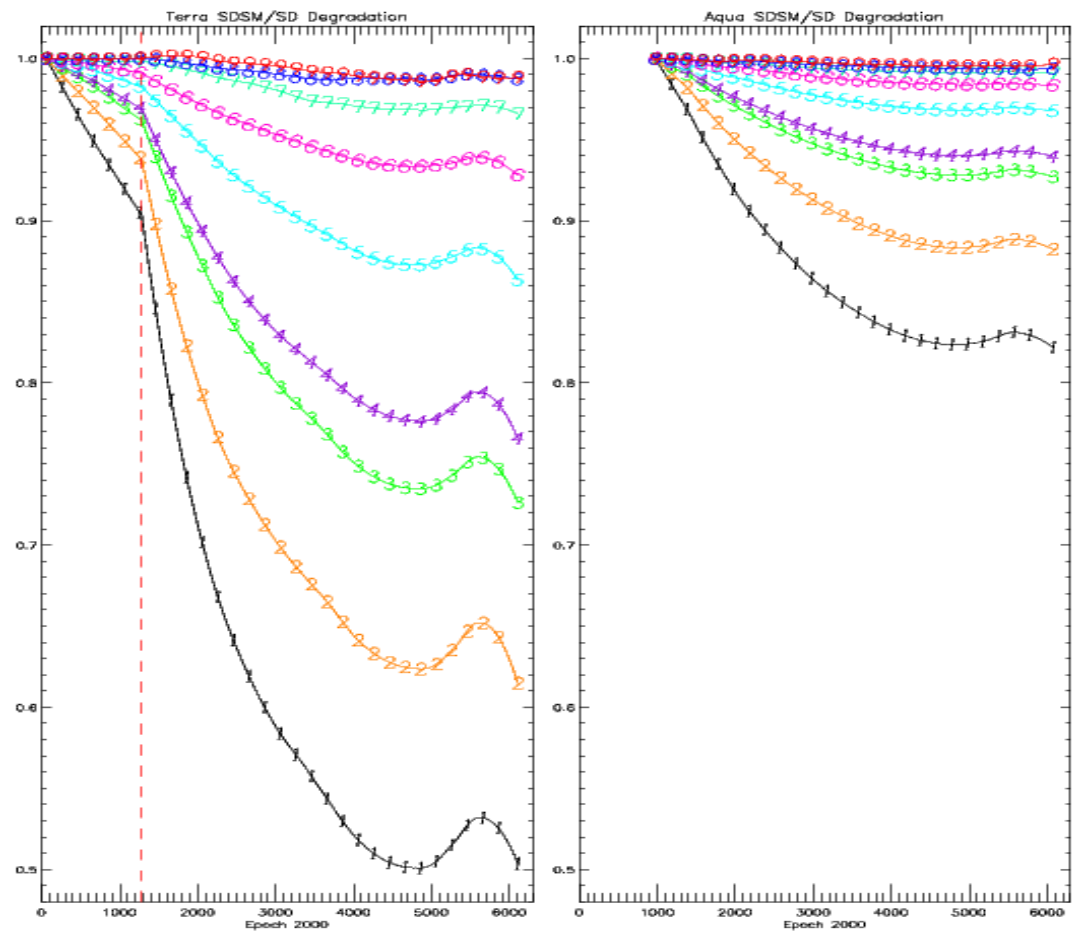

Figure 7 MODIS Terra and Aqua SDSM/SD degradation trending.

After MODIS Aqua/Terra degradation profiles are characterized over all nine SDSM detectors, the overall SD performance of MODIS Aqua and Terra can be evaluated. Using the relationship between SDSM detectors and RSB bands, and a linear interpolation approximation, the MODIS SD degradation versus center wavelength can be obtained. Figure 8 plots their yearly degradation performance. In the yearly-based considerations, Aqua SD degrades about $19 \%$ at the center wavelength of $412 \mathrm{~nm}$ in 14 years; however Terra SD degrades about $48 \%$ in 16 years. Notice that the degradation is somehow saturated in both instruments. The curve difference in the last 4 years is about $1.0 \%$ for Aqua, and $1.2 \%$ in the last 6 years for Terra. 

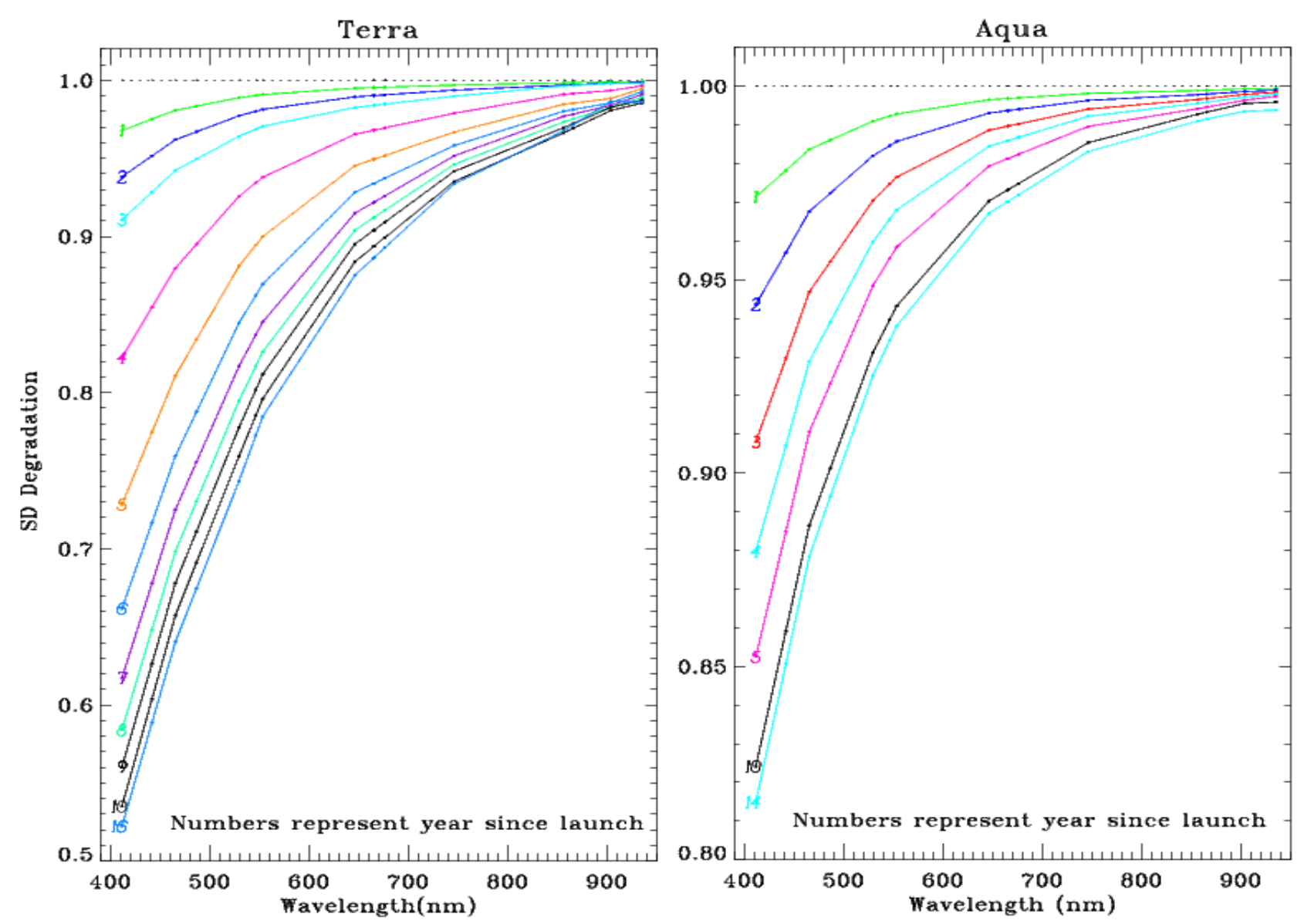

Figure 8 MODIS SD degradation. Colored curves indicate years since launch.

\section{CONCLUDING REMARKS}

In this paper, a recent improvement of MODIS SD on-orbit degradation characterization has been presented. The overall on-orbit performance of Aqua and Terra MODIS SD/SDSM has been evaluated. This LUT-based approach is extended to other detectors using a dynamic scheme. With the LUT based geometrical compensation, the degradation of all SDSM detectors is effectively estimated. By applying the LUT geometrical compensation, the SDSM observations can be consistently used to track the on-orbit changes of the SD BRF, such that high quality RSB calibration and science data quality are maintained. To combat the impact of SDSM Sun view ripples, different approaches have been discussed to track MODIS SD on-orbit degradation. Compared with the raw SDSM measurements, the dynamic LUT compensation out-performs the other options in terms of observed fluctuations, for both MODIS Aqua and Terra. Results of the SD degradation derived from SDSM measurements are presented and compared. Specifically, operating after about sixteen years of operation, the Terra MODIS SD BRF has a wavelength dependent degradation of $47.7 \%$ at $412 \mathrm{~nm}, 36.0 \%$ at $466 \mathrm{~nm}, 25.7 \%$ at $530 \mathrm{~nm}, 21.5 \%$ at $554 \mathrm{~nm}, 12.5 \%$ at $646 \mathrm{~nm}, 6.6 \%$ at $747 \mathrm{~nm}, 3.3 \%$ at $857 \mathrm{~nm}, 1.4 \%$ at $904 \mathrm{~nm}$ and $1.4 \%$ at 936nm. Meanwhile, in over fourteen years of operation, the degradation profile of Aqua MODIS SD BRF is $18.5 \%$ at $412 \mathrm{~nm}, 12.2 \%$ at $466 \mathrm{~nm}, 7.5 \%$ at $530 \mathrm{~nm}, 6.2 \%$ at $554 \mathrm{~nm}, 3.3 \%$ at $646 \mathrm{~nm}, 1.7 \%$ at $747 \mathrm{~nm}, 0.9 \%$ at $857 \mathrm{~nm}, 0.6 \%$ at $904 \mathrm{~nm}$ and $0.6 \%$ at $936 \mathrm{~nm}$. 


\section{ACKNOWLEDGEMENTS}

The authors would like to thank other members of the MODIS Characterization Support Team (MCST) for their technical discussions and assistance, especially Dr. Truman Wilson for his helpful comments.

\section{REFERENCES}

[1] V. Salomonson, W. Barnes, X. Xiong, S. Kempler, and E. Masuoka, "An overview of the Earth Observing System MODIS instrument and associated data systems performance", in Proc. IGARSS, 2002, pp.1174-1176

[2] J. Sun, X. Xiong and W.L. Barnes, "MODIS Solar Diffuser Stability Monitor Sun View Modeling", IEEE Trans. on Geosci. Remote Sens., 43(8), 1845-1854, 2005.

[3] H. Chen, Z. Wang, J. Sun, A. Angal and X. Xiong, "Recent Progree of MODIS Solar Diffuser On-orbit Degradation Characterization", proceedings of SPIE - Earth Observing Systems XVII, Vol. 8510, 2012.

[4] X. Xiong, J. Sun, W. Barnes, V. Salomonson, J. Esposito, H. Erives and B. Guenther, "Multiyear On-Orbit Calibration and Performance of Terra MODIS Reflective Solar Bands", IEEE Trans. on Geosci. Remote Sens., 45(4), 879-889, 2007.

[5] H. Chen and X. Xiong, "MODIS Solar Diffuser Monitor: Function and Applications", proceedings of SPIE - Earth Observing Systems XIV, Vol. 7452, 2009.

[6] X. Xiong, H. Erives, S. Xiong, X. Xie, J. Esposito, J. Sun and W. Barnes, "Performance of Terra MODIS Solar Diffuser and Solar Diffuser Stability Monitor", proceedings of SPIE - Earth Observing Systems X, Vol. 5882, 2005.

[7] J. Sun, X. Xiong and B. Guenther, "MODIS Solar Diffuser Stability Monitor Performance", proceedings of SPIE Earth Observing Systems VI, Vol. 4483, 156-164, 2002.

[8] X. Xiong, X. Xie, A. Angal, J. Choi, J. Sun and W. Barnes, "Characterization of MODIS Solar Diffuser On-orbit Degradation", proceedings of SPIE - Earth Observing Systems XII, Vol. 6677, 2007.

[9] X. Xiong, and W.L. Barnes, "An Overview of MODIS radiometric calibration and characterization", Adv. Atmos. Sci., 23(1), 69-79, 2006. 